\title{
Optimization of small satellite constellation design for continuous mutual regional coverage with multi-objective genetic algorithm
}

\author{
I. Meziane-Tani ${ }^{12}$, G. Métris ${ }^{2}$, G. Lion ${ }^{3}$, A. Deschamps ${ }^{2}$, F. T. Bendimerad ${ }^{1}$, M. Bekhti ${ }^{4}$ \\ ${ }^{1}$ Laboratoire de Télécommunications de Tlemcen, Faculty of Technology, University of Tlemcen, \\ BP 230, \\ Tlemcen, 13000, Algeria \\ E-mail: mezianetani.imane@gmail.com \\ ${ }^{2}$ Géoazur, Université de Nice Sophia Antipolis, CNRS (UMR 7329), Observatoire de la Côte d'Azur, \\ 250 rue Albert Einstein, \\ Sophia Antipolis, 06560, France \\ ${ }^{3}$ LNE-SYRTE, Observatoire de Paris, CNRS (UMR8630), \\ 61 Avenue de l'Observatoire, \\ Paris, 75014, France \\ ${ }^{4}$ Algerian Space Agency (ASAL), Centre of Satellite Development, \\ POS 50 ILOT T12 Bir El Djir, \\ Oran, 31000, Algeria
}

Received 22 March 2015

Accepted 6 March 2016

\begin{abstract}
This paper describes the application of an evolutionary optimization method to design satellite constellation for continuous regional coverage without intersatellite links. This configuration, called mutual coverage, is related to some technical limitations that exist on small satellite technology. The coverage of the north Algerian seismological network is taken as an example of application. A Multi Objective Genetic Algorithm (MOGA) is used to make a trade-off between the improvement of the coverage rate, the minimization of the total number of satellites and the reduction of the satellites' altitude. First, some experiments have been performed to find the weight distribution of the fitness function that shows the most significant improvement of the average fitness function. Then, some optimized constellation designs are given for different ranges of altitude and it is shown that the size of the MOGA constellation design is significantly reduced compared to the traditional geometrical design.
\end{abstract}

Keywords: Multi Objective Genetic Algorithm; Constellation Design; Regional Coverage; Mutual Coverage; Small Satellite Constellation

\section{Introduction}

Designing a constellation of satellites for a given space mission is a complex multi-parametric opti- mization process. The aim is to find a constellation with a minimum number of satellites, in order to reduce the system's cost and complexity. Besides the number of satellites, the designer has to

\footnotetext{
* E-mail: mezianetani.imane@gmail.com. Tel.: (+213) 432856 89; fax: (+213) 43285685
} 
define the configuration of the constellation by specifying the trajectory of each satellite defined by six initial Keplerian orbital elements: semi-major axis $a$, eccentricity $e$, inclination $i$, longitude of the ascending node $\Omega$, argument of perigee $\omega$ and mean anomaly $M$ (See e.g ${ }^{1}$ ).

In order to simplify the design process and to reduce the launch cost and scheduling, symmetrical constellations have been introduced $\left(\mathrm{See}^{2}\right)$. According to the type of coverage, some models exist based on geometrical approximations. For instance, the Delta patterns constellations proposed by Ballard ${ }^{3}$ and Walker ${ }^{4}$ are the most popular for single global coverage using circular orbits; while the Street-of-coverage geometry introduced by Lüders in $19611^{5}$ is used for single and redundant regional coverage by Rider ${ }^{6,7}$. However, some missions requiring specific coverage constraints need to be resolved by means of optimization methods ${ }^{8}$.

Evolutionary algorithms like simulated annealing ${ }^{9}$, Ant colony ${ }^{10}$ and Genetic Algorithms (GA) ${ }^{11}$ have been used to propose new satellite constellation geometries and to help for the optimization of existing constellation designs. Most of the applications have studied the global coverage general case. We can cite Ferringer, et al. ${ }^{12}$ and Whittecar, et. al ${ }^{13}$.

For a regional coverage problem, which is our object of study, Genetic Algorithms have been mostly employed compared to other methods. In 2001, Confessor, et al. ${ }^{14}$ proposed a genetic algorithm to design an elliptical orbit constellation for regional coverage and bands coverage in high latitude regions. The regional coverage has been also deeply explored in the region of China by Wang, et al. ${ }^{15}$, Mao, et al. ${ }^{16}$ and Xiao, et al. ${ }^{17}$ to design constellations increasingly optimal for different mission objectives (communication, navigation, etc.).

In this work, we introduce the design optimization process of constellations of small satellites for regional coverage without intersatellite links (ISLs). As an example, we propose the application of regional data collection. In traditional communication satellite networks, when the data center is not visible from the satellite forwarding the data, the latter is routed via other intermediate satellites by means of intersatellite links until it reaches the data center.
Small satellites and especially the CubeSats ${ }^{18}$ used in this study present some technical limitations. One of them supposes that it is difficult to control ISL because of the low accuracy of the attitude control subsystem ${ }^{19}$. Therefore, in order to maintain a continuous coverage of the ground stations and the data center, we consider that the satellites must insure continuously a mutual coverage of all the ground stations and the data center supposed to be within the same area.

For this purpuse, this paper is organized as follows: first, the concept of mutual coverage is described in Section 2. Due to the fact that the existing geometrical models do not solve the mutual coverage design problem and most of them treat the instantaneous coverage of a single point on Earth, Section 3 is intended to describe the implementation of a genetic algorithm to design a constellation of satellites dedicated to provide a mutual coverage of a given region. Because we shall take into account many objectives (visibility, number of satellites, orbital altitude) in the fitness function, we are using a multi objective genetic algorithm, called MOGA (for more details, see e.g. ${ }^{20},{ }^{21}$ ).

Section 4 presents an example of application and describes the design of the area considered for the mutual regional coverage, which is the north Algerian seismological network, as well as the selected GA parameters. Finally, the results are presented and discussed in Section 5 where some optimal constellation designs are computed with the help of the GA. The results obtained are compared with the traditional geometrical approach used in single point coverage.

\section{Constellation geometry for regional coverage}

\subsection{Continuous coverage}

The main objective in permanent data collection is to maintain a continuous coverage of the region of interest. Because of the reduced time of visibility due to the Earth's rotation, a constellation organized into $P>1$ orbital planes, each containing $s$ satellites, is needed in Low Earth Orbits (LEO). For illustration, Figure 1 shows a $3 \mathrm{D}$ view of a constellation of 
inclined LEO satellites with 9 orbital planes, each containing 4 satellites.

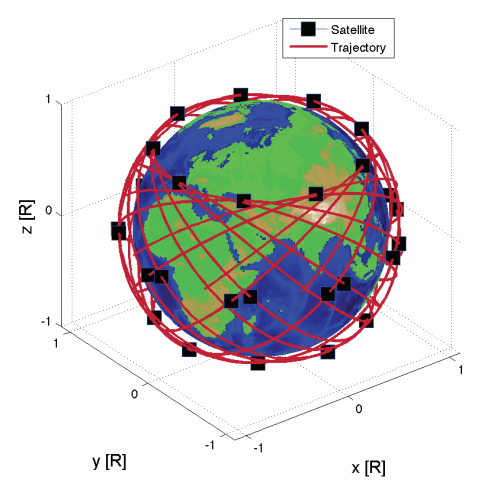

Fig. 1. 3D view of a LEO satellite constellation $(P=9, s=$ 4). Filled squares: the positions of the satellites. R: the Earth's mean radius.

Near-circular orbits $(e \simeq 0)$ are more suitable for communication satellites because the satellite is at a nearly constant altitude resulting in a constant strength signal to communicate. The $P$ orbital planes are chosen with the same inclination angle and regularly distributed in longitude of the ascending node $\Omega$. In what follows, the altitude parameter $h$ is used instead of the semi-major axis $a$, with $a=R+h$ and $R \simeq 6371 \mathrm{~km}$ the Earth's mean radius. $h$ is considered to be constant for all the satellites. When considering all these simplifications, Figure 2 illustrates the geometrical coverage configuration of a single point on Earth by a satellite orbiting at an altitude $h$ of a circular orbit.

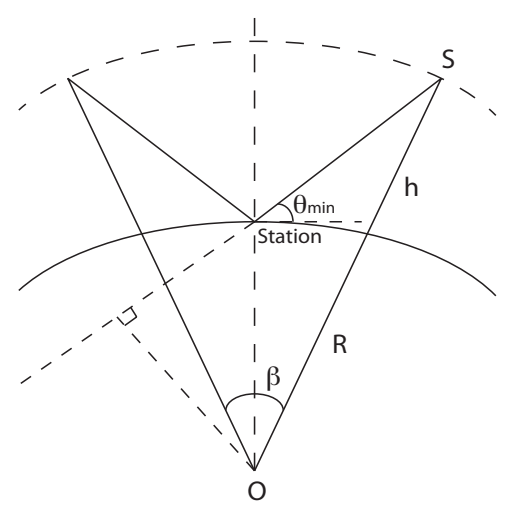

Fig. 2. Coverage geometry of a satellite with a conical fieldof-view.
For a constellation covering permanently this point, the number of orbital planes, $P$, and the number of satellites per orbital plane, $s$, can be deduced by the expressions,

$$
\begin{gathered}
P_{\text {min }}=\left\lceil\frac{2 \pi}{\beta}\right\rceil \\
s_{\text {min }}=\left\lceil\frac{\pi}{\beta}\right\rceil
\end{gathered}
$$

The symbol \lceil\rceil denotes the ceiling function and $\beta$, the Earth's central angle of visibility viewed from its center, is defined by

$$
\beta=2 \arccos \left(\frac{R}{R+h} \cos \left(\theta_{\min }\right)\right)-\theta_{\min }
$$

with $\theta_{\min }$ the minimum elevation angle. From ${ }^{2}$, it is supposed that the minimum elevation angle $\theta_{\min }$ must be typically equal to $5^{\circ}$ for satellite-station communications.

\subsection{Mutual coverage}

Intersatellite links are two way communication paths between satellites. They are used to increase the temporal resolution of LEO satellites. However, in order to maintain the communication between two satellites, a high accuracy of the satellite attitude control is required, and because of the relative motion between the satellites, the use of an antenna steering mechanism is unavoidable. All these requirements will make ISL difficult to perform on a simply designed CubeSat as it is supposed here. Thus, we consider that no ISLs are available, meaning that a mutual coverage has to be maintained between all the transmitting stations and the data center.

A satellite mutual coverage of two different Earth stations means that at instant $t$, a satellite has to be visible from both stations (in our case a transmitting station and the data center DC) as illustrated in Figure 3. 


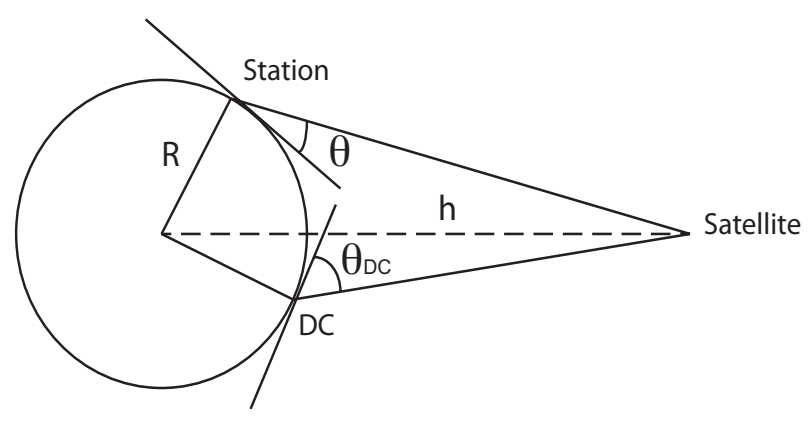

Fig. 3. Mutual coverage of two ground stations from a satellite point of view.

The coverage function from the $i$-th satellite to the $j$-th station and the data center (DC) at the instant $t$ is defined by

$c_{i, j, D C}(t)= \begin{cases}1 & \text { if } \theta_{i, j}(t) \geqslant \theta_{\text {min }} \text { and } \theta_{i, D C}(t) \geqslant \theta_{\text {min }} \\ 0 & \text { else }\end{cases}$

where $\theta_{i, j}(t)$ and $\theta_{i, D C}(t)$ are the elevation angles of the $i$-th satellite from the $j$-th station and the data center DC, respectively. We suppose that a constellation with $N$ satellites is visible if at least one satellite is visible. Then, the coverage of the constellation from station $j$ and the DC at an instant $t$ is defined as

$$
C_{j, D C}(t)=\max _{1 \leqslant i \leqslant N} c_{i, j, D C}(t)
$$

\section{Constellation design using Genetic Algorithm}

Genetic algorithms (GA) proved their effectiveness as an optimization method for nonlinear multiparametric problems ${ }^{22}$. Starting with a random intial population of solutions, a GA selects individuals with good chances of reproduction (best fitness function) and reproduces the new generation of individuals using operations such as crossover and mutation. The process is repeated several times until it runs a certain number of generations or until a solution considered as optimum is reached.

The parameters of an individual to be optimized are represented by a structure called chromosome and a binary coding is adopted here.

For satellite constellation design each chromosome represents a constellation model and the parameters to be optimized are the constellation parameters $P$ and $s$, the altitude $h$ and the inclination angle $i$. For the other orbital parameters, according to the simplifications supposed before, the orbital eccentricity $e=0$ and the argument of perigee $\omega=0 . \Omega$ and $M$ are uniformly distributed for all the constellation. The aim of our optimization is to design a constellation which offers a continuous mutual coverage of a region by reducing the constellation size (and consequently the system cost) and the orbital altitude of the satellites. To reduce the CubeSat's altitude is interesting in order to find easily launch opportunities and also to respect the lifetime restriction law. This point will be discussed later with more details.

A Multi Objective Genetic Algorithm (MOGA) is then implemented to find a trade-off between the following three objectives:

(i) Maximize the visibility rate, $R v$;

(ii) Minimize the total number of satellites, $N$;

(iii) Reduce the altitude, $h$.

\subsection{Fitness evaluation}

Every individual (constellation) is assigned a value called fitness which is used to select the elected individuals for reproduction. The fitness function takes into account the visibility rate, the total number of satellites $N$ and the altitude $h$. We apply a traditional weighted multi objective function ${ }^{23}$. Thus, the fitness function of a constellation $i$ can be expressed as:

$$
f_{i}=w_{R v} \times R v_{i}+w_{N} \times \frac{N_{\min }}{N_{i}}+w_{h} \times \frac{h_{\min }}{h_{i}}
$$

where $w_{R v}, w_{N}$ and $w_{h}$ are the weights associated with the importance of each objective function and $w_{R v}+w_{N}+w_{h}=1 . N_{i}$ and $h_{i}$ are the size and the altitude of the constellation $i$. To normalize and increase the fitness function, $N_{\min }$ and $h_{\min }$ are the minimum values of $N$ and $h$ in the search range. $R v_{i}$ is the visibility rate of a constellation $i$. It is defined by the mean of the visibility rates of the $m$ stations we would like to cover mutually with respect to the data center:

$$
R v_{i}=\frac{\sum_{j=1}^{m} R_{j}}{m}
$$


where, $R_{j}$, the visibility rate of a station $j$ during a period $T$ is given by:

$$
R_{j}=\frac{D V_{j, D C}}{T}
$$

and $D V_{j, D C}$ is the mutual visibility duration of a constellation from station $j$ and the data center during period $T$. It is deduced from equation (4) and is given by:

$$
D V_{j, D C}=\int_{t_{0}}^{t_{0}+T} C_{j, D C}(t) \mathrm{d} t
$$

\subsection{Selection}

The individuals are selected according to their probability of reproduction. Among the different selection methods that exist ${ }^{24}$, we decided to use a random selection that simulates a roulette wheel with fields of different sizes. Each individual is associated to a field. The size of the field is proportional to its fitness (higher fitness: bigger fields; lower fitness: smaller fields).

In a population of size $N_{p}$, the probability of selection of an individual with index $i$ is

$$
P(i)=\frac{f_{i}}{S}
$$

where $S=\sum_{j=1}^{N_{p}} f_{j}$. Then, the $i-t h$ individual is selected if it satisfies the condition

$$
\sum_{j=1}^{i} f_{j}>r
$$

with $r$ a random number bewteen 0 and $S$.

This selection process is then repeated until the number of selected individuals equals the population size.

\subsection{Reproduction}

To create new offspring from the selected parents, two main operations are usually used.

(i) Crossover: New offspring which share the parents' genetic inheritance (genes) are generated with probability $P_{c}$. (usually $0.1 \leqslant P_{c} \leqslant 0.9$ ); (ii) Mutation: This allows for diversity in the population by generating new types of genes in the chromosomes. This operation is less frequent than crossover and occurs on a chromosome's bits with a probability $P_{m} \leqslant 0.05$.

\section{Satellite constellation simulation results}

\subsection{Coverage parameters}

As an example of regional data collection application, we consider the Algerian seismological network deployed in the northern part of the country. This area is located between latitudes $33^{\circ} \mathrm{N}$ and $37^{\circ} \mathrm{N}$ and longitudes $-2^{\circ} \mathrm{E}$ and $8^{\circ} \mathrm{E}$, and the data center $(\mathrm{DC})$ is situated at $\left(36.7^{\circ} \mathrm{N} ; 3.02^{\circ} \mathrm{E}\right)$.

In order to simplify the coverage geometry, the area to be covered is represented by the dashed polygone covering the whole ground network as it is illustrated in Figure 4. Table 1 contains the geographical coordinates of the 5 positions $(\mathrm{A}, \mathrm{B}, \mathrm{C}, \mathrm{D}, \mathrm{E})$ surrounding this area and the position of the $\mathrm{DC}$.

We suppose that, at an instant $t$, each couple of points [DC-A, DC-B, DC-C, DC-D, DC-E] is covered by at least one satellite. In addition, if a satellite simultaneously covers the DC and one of the points, then all the stations located within this axis are also visible by this satellite.

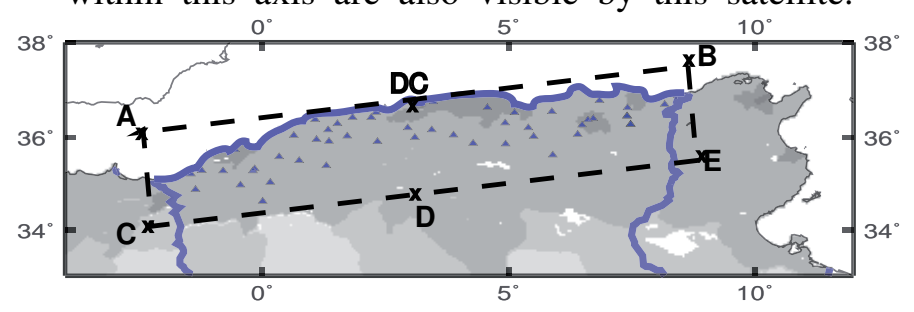

Fig. 4. Geographical delimitation of the coverage of the north Algerian seismological network.

Table 1. Positions of the terrestrial limits (A, B, C, D, E) of the area to be covered as well as the data center DC.

\begin{tabular}{c|c|c}
\hline & Latitude $\left(^{\circ}\right)$ & Longitude $\left(^{\circ}\right)$ \\
\hline DC & 36.7 & 3.02 \\
A & 36.32 & -2.51 \\
B & 37.43 & 8.52 \\
C & 33.67 & -2.10 \\
D & 34.22 & 3.41 \\
E & 34.78 & 8.93 \\
\hline
\end{tabular}




\subsection{Genetic algorithm parameters}

Since we consider only symmetrical constellations, inclined near-circular orbit constellations are designed in this study. Four parameters are to be optimized: the number of orbital planes $P$, the number of satellites by orbital plane $s(P \times s=N)$, the orbit altitude $h$ and the inclination angle $i$. In order to avoid the radiation emitted by the Van Allen belt which may affect the CubeSat components, the satellites are in general launched at altitudes below $1000 \mathrm{~km}$. Furthermore, in order to reduce the atmospheric drag and thus increase the mission lifetime, we put the minimum altitude at $500 \mathrm{~km}$. The orbit inclination angle is usually chosen close to the maximum latitude of the area to be covered which is bounded by the limits presented in Table 1 .

Hence, the constraints on the altitude $h$ and inclination angle $i$ are

$$
500 \mathrm{~km} \leqslant h \leqslant 1000 \mathrm{~km}, \quad 37^{\circ} \leqslant i \leqslant 50^{\circ}
$$

and the selected parameters of the GA are

$$
\begin{aligned}
& N_{\text {Population }}=30, \quad N_{\text {Generation }}=200, \\
& P_{C}=0.5, \quad P_{m}=0.01 .
\end{aligned}
$$

$P_{m}=0.01$ is a typical value. $P_{C}=0.5$ has been chosen because when compared with $P_{C}=0.9$ (the value of $P_{C}$ in Non-dominated Sorting Genetic Algorithm, NSGA-II ${ }^{25}$ ) it shows a better evolution of the average fitness function, which is an evaluation parameter that we are discussing in Section 5. The values of $N_{\text {Population }}$ and $N_{\text {Generation }}$ are sufficient for the evaluation below.

The fitness function, defined previously in equation (5), uses the mean value of visibility rate of the mutual coverage between the data center and the positions (A, B, C, D, E). To do that, we have implemented a near-circular orbit propagation simulator using the software Matlab. The effect of the Earth's dynamical flattening linked to the Stokes coefficients $J_{2}$ is the main perturbation. Our software evaluates the visibility time of the satellites of the constellation from each couple of ground stations during a period $T=24$ hours.

Also, the time sampling is chosen to be 30 seconds. Besides the fact that this choice will reduce the calculation time of our algorithm, it is considered that the occurrence of 30 seconds gaps in the data transmission is not critical in the case of seismological monitoring process. A constellation is considered best when its fitness function is higher than the fitness of the other constellations in the population. To fit perfectly with the continuous mutual coverage objective, the selected constellation must have $100 \%$ of visibility rate.

\section{Results and Discussion}

\subsection{Weights distribution of the fitness function}

One of the most important choices that affects the GA evolution is the fitness function. The average fitness function (AFF) has to be improved during the optimization process. In Figure 5, we have tested different weight distributions, defined in (5), to maximize the AFF with respect to the number of generations. One way to do that is to choose a weight distribution leading to a good evolution of the GA. According to the importance of each objective function, we have considered that the weight associated to the maximization of the visibility rate, $w_{R v}$, is always greater than $w_{N}$ and $w_{h}$ : the weights for the minimization of $N$, and $h$, respectively. To normalize the fitness function, we have put $N_{\min }=12$ and $h_{\text {min }}=500 \mathrm{~km}$.

We can see in Figures 5a, $5 \mathrm{~b}$ and $5 \mathrm{c}$ that the AFF trend decreases for $w_{R v} \leqslant 0.6$. However, an improvement is observed when $w_{R v}$ is much greater than $w_{N}$ and $w_{h}$ for $w_{R v} \geqslant 0.7$. This improvement is especially more significant for the weight distribution $\left(w_{R v}=0.9, w_{N}=0.05, w_{h}=0.05\right)$ (Figure 5f). For that reason, we shall use this weight distribution in what follows.

Figure 6 shows the set of solutions with $R v=100 \%$ where the number of satellites $N$ is plotted versus the altitude $h$. 


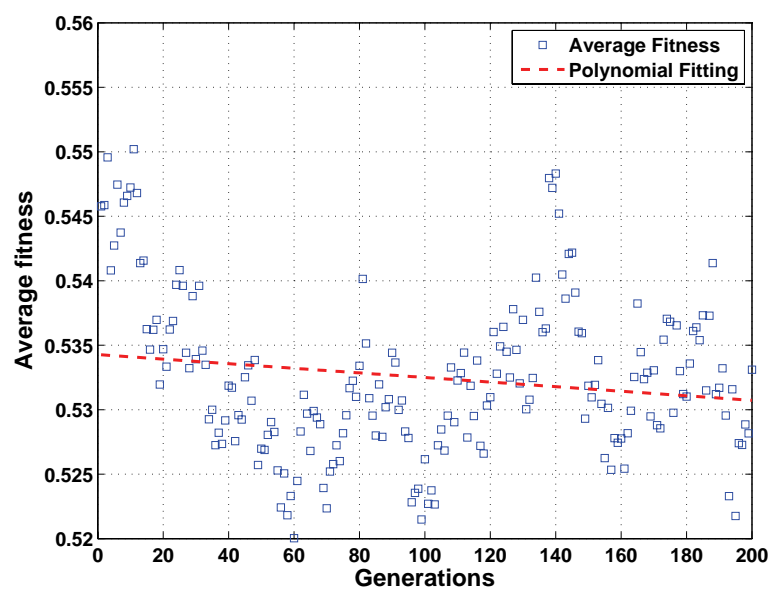

(a) $w_{R V}=0.4, w_{N}=0.4, w_{h}=0.2$.

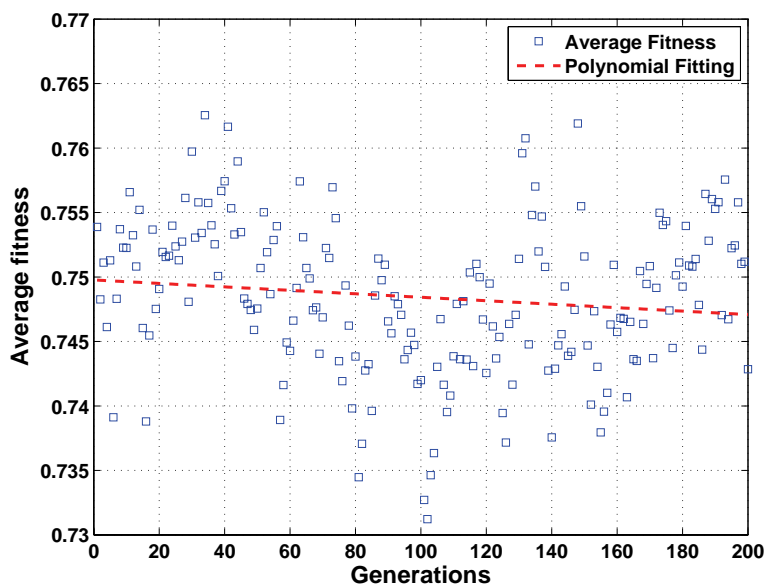

(c) $w_{R V}=0.6, w_{N}=0.25, w_{h}=0.15$.

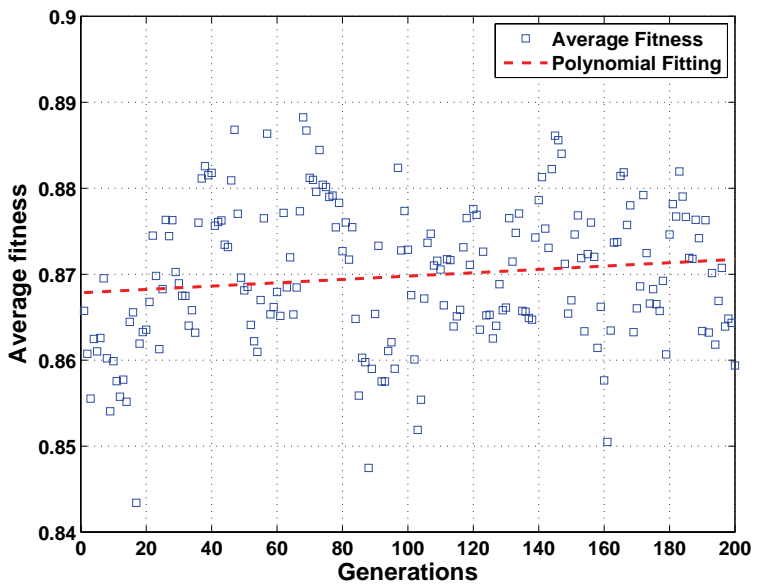

(e) $w_{R V}=0.8, w_{N}=0.1, w_{h}=0.1$.

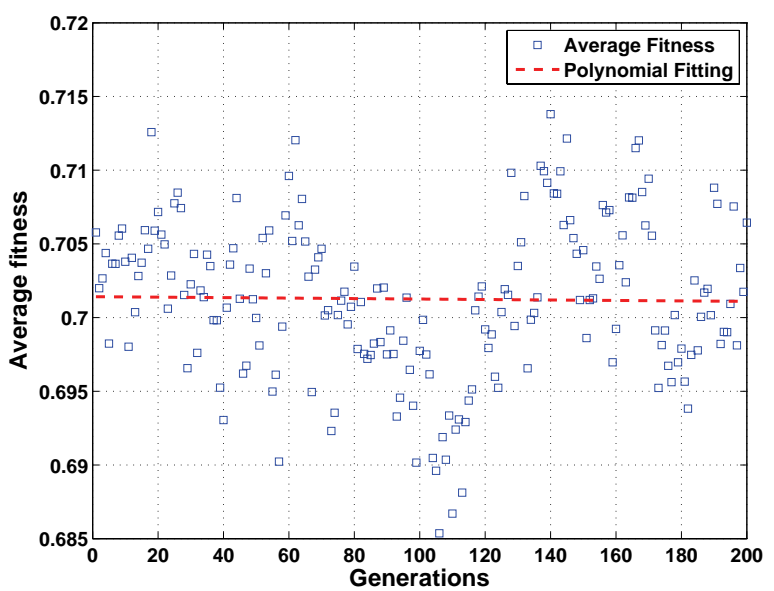

(b) $w_{R V}=0.5, w_{N}=0.3, w_{h}=0.2$.

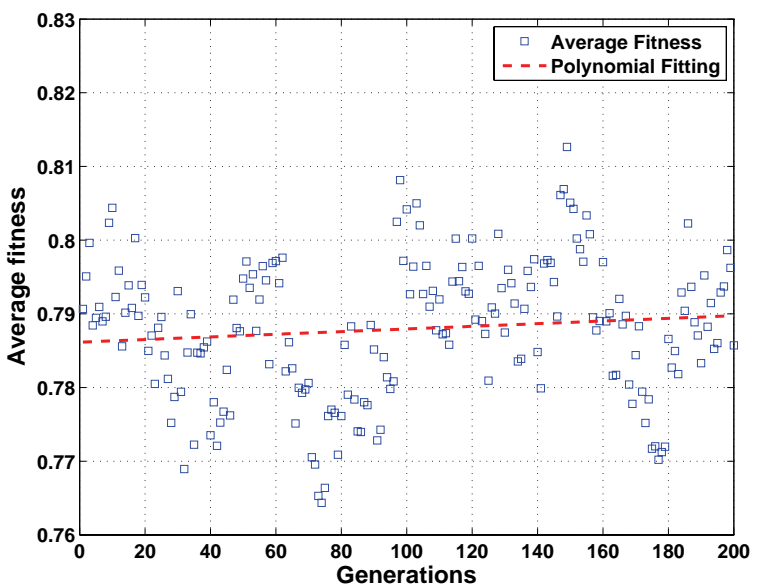

(d) $w_{R V}=0.7, w_{N}=0.2, w_{h}=0.1$

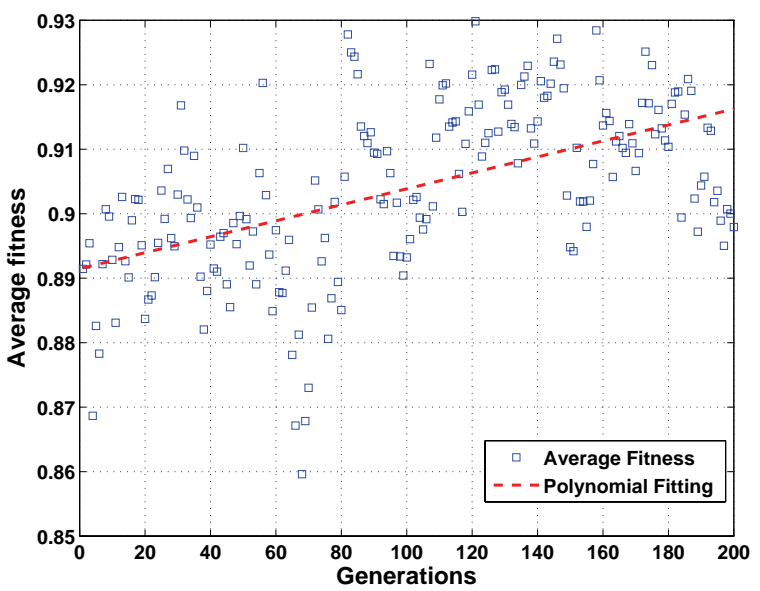

(f) $w_{R V}=0.9, w_{N}=0.05, w_{h}=0.05$.

Fig. 5. Evolution of the AFF during 200 generations for different weight distributions. 


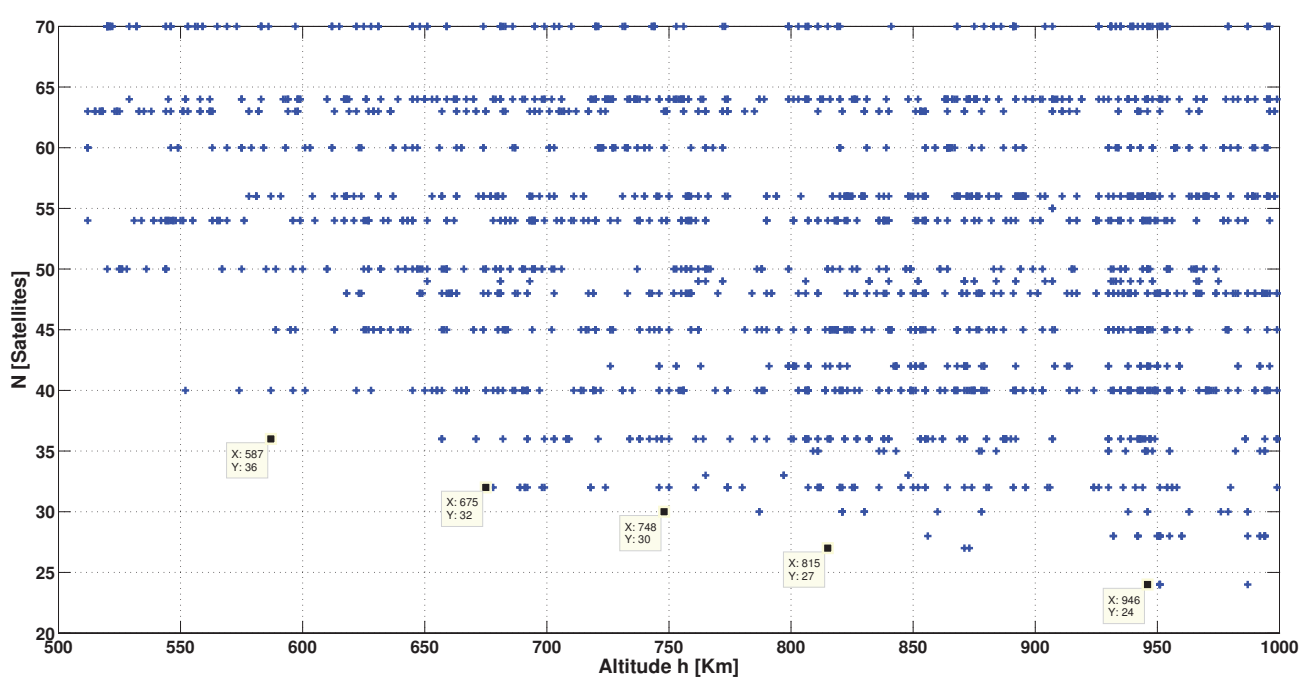

Fig. 6. $N$ versus $h$ of constellations with $100 \%$ of visibility rate. The black squares with labels show the solutions with the minimum $N$ and the minimum $h$ in each interval of altitudes.

Since we are interested in constellations with the minimum $N$ and the lowest altitude $h$, we splitted the interval of altitudes (500-1000 km) into 5 subinter- vals. The minimum $N$ we obtained for each interval and the corresponding altitude are given in Table 2.

Table 2. Minimum $N$ and $h$ for each interval of altitudes.

\begin{tabular}{lccccc}
\hline$h$ interval $[\mathrm{km}]$ & {$[500-600]$} & {$[600-700]$} & {$[700-800]$} & {$[800-900]$} & {$[900-1000]$} \\
\hline Minimum $N$ & 36 & 32 & 30 & 27 & 24 \\
Minimum $h[\mathrm{~km}]$ & 587 & 675 & 748 & 815 & 946 \\
\hline
\end{tabular}

\subsection{Optimization}

In a practical case, when designing a constellation of satellites we are faced with certain constraints. Some of them are related to the budget that mainly limits the maximum number of satellites, $N$, and others are related to the selected launcher and orbits to be reached (particularly $h$ and $i$ ). For that reason, we suppose that the maximum number of satellites and the maximum altitude are fixed. There is no constaint on the inclination angle since it has not been taken into account in the optimization objectives.
The aim of this step is to improve the results obtained in Table 2. The MOGA is used to find and design an optimum constellation by taking into account these contraints:

- $R v=100 \%$

- Constellation size $N \leqslant N_{\max }$;

- Altitude of the satellites orbit $h \leqslant h_{\max }$.

This approach is repeated for the other conditions in Table 2 and the results are summarized in Table 3. 
I. Meziane-Tani et al. / Optimization of small satellite constellation design

Table 3. Local optimum satellite constellations whith two requirements: $N_{\max }$ and $h_{\max }$.

\begin{tabular}{lc|ccccc|cc}
\hline \multicolumn{1}{l|}{ Expected solution } & \multicolumn{6}{|c|}{ Solution found } & \multicolumn{2}{c}{$\begin{array}{l}\text { Geometrical } \\
\text { design }\end{array}$} \\
\hline$N_{\max }$ & $h_{\max }[\mathrm{km}]$ & $N$ & $h[\mathrm{~km}]$ & $P$ & $i[\mathrm{deg}]$ & Number of Generations & $N$ & $P$ \\
\hline 36 & 587 & 36 & 584 & 9 & 42 & 7 & 60 & 10 \\
32 & 675 & 30 & 672 & 10 & 48 & 46 & 54 & 9 \\
30 & 748 & 30 & 705 & 10 & 49 & 24 & 45 & 9 \\
27 & 815 & 27 & 812 & 9 & 50 & 13 & 40 & 8 \\
24 & 946 & 24 & 928 & 8 & 48 & 12 & 40 & 8 \\
\hline
\end{tabular}

The first part of Table 3, "Expected solution", contains $N_{\max }$ and $h_{\max }$. In our case, because we know in advance the expected solution, this one is used as a stop condition of the GA. Thus, we have chosen the expected solutions $\left(N_{\max }, h_{\max }\right)$ from the results obtained in the first part of the optimization. Thus we know in advance that these solutions exist. However in a practical case, we have a couple of conditions $\left(N_{\max }, h_{\max }\right)$ and we try to verify if an optimal solution can be found with the MOGA. If so, the constellation parameters $(N, P, h, i)$ are given by the algorithm. Otherwise we conclude that no solution has been found after a certain number of generations.

The second part of Table 3, "Solution found", presents the results of the optimization process and gives the parameters of the designed constellation $(N, h, P, i)$ as well as the number of generations spent to find this solution. We notice that, in all the simulations, the altitude $h$ has been slightly reduced which probably means that the results obtained from the first part of the study was near to the optimum and that $N_{\text {Generation }}=200$ was enough to carry out the optimization. Also, we notice that for the solution of the second simulation (second line of the Table 3), $N$ has been reduced from 32 to 30 satellites. For the other cases, the optimization gives the same results, which means again that the results presented in Table 2 are effective.

The last part of Table 3, "Geometrical design", serves to compare the optimized constellation parameters with the constellation that would be designed with a simple geometrical approach presented in Section 2.1 for continuous coverage design. We notice that the constellation size $N$ is sig- nificantly optimized using the GA compared to a traditional geometrical approach. Indeed, for an altitude equal to $584 \mathrm{~km}$, the minimum $N$ found with the GA optimization is 36 satellites while it is 60 with a geometrical design. The gain of $N$ is about $40 \%$. This difference is due to the approximations supposed in the geometrical configuration (represented in Figure 2) such as the spherical aspect of the Earth (the Earth's radius $R$ is considered constant at every point on Earth). However, the effect of the Earth's dynamical flattening, $J_{2}$, has been considered in the orbital simulation used by the GA optimization.

\subsection{Satellite orbital lifetime}

For continuous mutual coverage of the north Algerian seismological network, the minimum constellation designed using our MOGA corresponds to the last line of Table 3: $N=24, h=928 \mathrm{~km}, P=8$ and $i=48^{\circ}$. However, satellites launched at these altitudes have an orbital lifetime greater than what it is allowed by the Inter-Agency Space Debris Coordination Committee (IADC) ${ }^{26}$. Indeed, for LEO satellites the orbital lifetime is limited to 25 years. Otherwise, the satellite must contain a deorbiting device ${ }^{27}$ which forces its re-entry. The use of such a system will increase the system cost and complexity. For that reason it is more suitable to opt for a solution without deorbiting system by choosing an appropriate orbital altitude.

The orbital lifetime of a CubeSat, with and without an on-board deorbiting device, is illustrated in Appendix A.1 for altitudes below $1000 \mathrm{~km}$. For altitudes up to $667 \mathrm{~km}$, we can see that the orbital lifetime of a satellite without a deorbiting device is 25 
years. Then, the optimum solution corresponding to this altitude is the constellation for which $N=36$ satellites, $P=9, i=42^{\circ}, h=584 \mathrm{~km}$.

\section{Conclusions}

In this paper we have presented some numerical results for designing a small satellite constellation using MOGA, intended to insure a coninuous mutual coverage of the north Algerian seismological network by reducing, precisely, the constellation size and the orbital altitude. Effects of the weight distribution of the fitness function have been studied and it appears that $\left(w_{R v}=0.9 ; w_{N}=0.05 ; w_{h}=0.05\right)$ is a good choice. Afterward, the algorithm has been used to design a set of constellations in a range of altitudes from 500 to $1000 \mathrm{~km}$ and according to some supposed project requirements: maximum total number of satellites and maximum altitude, and by taking into account the LEO orbital lifetime law, we can propose two solutions:

- Simple CubeSat: $N=36, P=9, i=42^{\circ}$ and $h=584 \mathrm{~km}$;

- CubeSat with deorbiting device: $N=24, P=8$, $i=48^{\circ}$ and $h=928 \mathrm{~km}$.

These first results are satisfying compared to a traditional geometrical design (60 satellites with geometrical design and 36 with the MOGA for $h=$ $584 \mathrm{~km}$ and $i=42^{\circ}$ ). However, further studies should be done to compare and improve these results. Testing other kinds of MOGA such as Randomly Assigned Weighted Aggregation (RAWA) or Non-dominated Sorting Genetic Algorithm (NSGAII $^{25}$ ) seems to be more pertinent than studying extensively the effects of other GA parameters (population size, generation size, crossover and mutation probability, etc.).

\section{Acknowledgments}

The authors are grateful to Averroes - Erasmus Mundus 2012, a Euro-Maghrebian exchange program for financially supporting this study which has been partially conducted in the laboratory Géoazur of the University of Nice Sophia-Antipolis, France.

\section{References}

1. M. Capderou. Satellites: Orbits and Missions. Springer, 2005.

2. J R Wertz. Mission geormetry : orbit and constellation design and management. El Segundo, California: Microcosm Press, 2001.

3. A. H. Ballard. Rosette constellations of earth satellites. Aerospace and Electronic Systems, IEEE Transactions on, AES-16(5):656-673, 1980.

4. J. G. Walker. Satellite constellations. Journal of the British Interplanetary Society, 37:559-572, December 1984.

5. R. D. Lüders. Satellite networks for continuous zonal coverage. ARS Journal, 31(2):179-184, 2013/11/20 1961.

6. L. Rider. Optimized polar orbit constellations for redundant earth coverage. Journal of the Astronautical Sciences, 33:147-161, June 1985.

7. L. Rider. Analytic design of satellite constellations for zonal earth coverage using inclined circular orbits. Journal of the Astronautical Sciences, 34:31-64, mar 1986.

8. E. Grandchamp and V. Charvillat. Metaheuristics to design satellite constellation. In Metaheuristics International Conference, pages 505-510. Citeseer, 2001.

9. W. A. Crossley and E. A. Williams. Simulated annealing and genetic algorithm approaches for discontinuous coverage satellite constellation design. Engineering Optimization A35, 32(3):353-371, 2000.

10. Q. He and Ch. Han. Satellite constellation design with adaptively continuous ant system algorithm. Chinese Journal of Aeronautics, 20(4):297-303, 2007.

11. E. Frayssinhes. Investigating new satellite constellation geometries with genetic algorithms. In Proceedings of the AIAA/AAS Astrodynamics Conference, pages 582-588, 1996.

12. Matthew P. Ferringer and David B. Spencer. Satellite constellation design tradeoffs using multiple-objective evolutionary computation. Journal of Spacecraft and Rockets, 43:1404-1411, 2006.

13. W. R. Whittecar and M. P. Ferringer. Global Coverage Constellation Design Exploration Using Evolutionary Algorithms. American Institute of Aeronautics and Astronautics, 2014.

14. G. Confessore, M. Di Gennaro, and S. Ricciardelli. A genetic algorithm to design satellite constellations for regional coverage. In Operations Research Proceedings, pages 35-41. Springer, 2001.

15. L. Wang, Y. Wang, K. Chen, and H. Zhang. Optimization of regional coverage reconnaissance satellite constellation by nsga-ii algorithm. In Information and Automation, 2008. ICIA 2008. International Conference on, pages 1111-1116, June 2008. 
16. T. Mao, Z. Xu, and R. Hou. Efficient constellation design based on improved non-dominated sorting genetic algorithm-ii. Journal of Computers, 7(6):13371344, 2012.

17. B. Xiao, Y. Liu, and G. Dai. Improved nsga-ii algorithm and its application in optimization of satellite constellation. Computer Engineering and Applications, 48(10):47, 2012.

18. R. Munakata et al. Cubesat design specification rev. 12. The CubeSat Program, California Polytechnic State University, 1, 2009.

19. J. Bouwmeester and J. Guo. Survey of worldwide pico-and nanosatellite missions, distributions and subsystem technology. Acta Astronautica, 67(7):854$862,2010$.

20. Abdullah Konak, David W Coit, and Alice E Smith. Multi-objective optimization using genetic algorithms: A tutorial. Reliability Engineering \& System Safety, 91(9):992-1007, 2006.

21. Tadahiko Murata, Hisao Ishibuchi, and Hideo Tanaka. Multi-objective genetic algorithm and its applications to flowshop scheduling. Computers \& Industrial Engineering, 30(4):957-968, 1996.

22. D. E. Goldberg. Genetic algorithms in search, optimization, and machine learning. Number 2. AddisonWesley, Reading, MA, 1989.

23. T. Murata and H. Ishibuchi. Moga: multi-objective genetic algorithms. In Evolutionary Computation, 1995., IEEE International Conference on, volume 1, pages 289-, Nov 1995.

24. D. E. Goldberg and K. Deb. A comparative analysis of selection schemes used in genetic algorithms. Urbana, 51:61801-2996, 1991.

25. Kalyanmoy Deb, Amrit Pratap, Sameer Agarwal, and TAMT Meyarivan. A fast and elitist multiobjective genetic algorithm: Nsga-ii. Evolutionary Computation, IEEE Transactions on, 6(2):182-197, 2002.

26. Inter-Agency Space Debris Coordination Committee IADC. Iadc space debris mitigation guidelines. 2007. URL: http://www.iadc-online.org [Last seen 2014-11-01].

27. E. Roberts, C. Peter, and Patrick G. Harkness. Drag sail for end-of-life disposal from low earth orbit. Journal of Spacecraft and Rockets, 44(6):1195-1203, 2014/04/13 2007.

28. STELA. Stela version 2.5.1, 2013. URL: http:// logiciels.cnes.fr/STELA/en/logiciel.htm [Last seen 2014-11-01].

29. C. Lücking. A passive high altitude deorbiting strategy. 25 th AIAA/USU Conference on Small Satellites, Logan, Utah, USA, 2011.

\section{Appendix A}

\section{A.1. Satellite lifetime estimation}

The Inter-Agency Space Debris Coordination Committee (IADC) ${ }^{26}$ limits the LEO satellites lifetime to 25 years. Numerical simulations have been performed using the software STELA ${ }^{28}$ to estimate the orbital end-of-life of a CubeSat $(10 \times 10 \times 10$ $\mathrm{cm}^{3}, 1 \mathrm{~kg}$ ) to be launched in the range of altitudes $(500-1000 \mathrm{~km})$ and with an orbit inclination angle equal to $41^{\circ}$. Figure A.1 shows the orbital lifetime of the selected CubeSat without and with a deorbiting device (marqued with *). The deorbiting device used is a passive drag sail ${ }^{27,29}$ with the dimensions presented in Table A.1. It is supposed to be deployed after the mission lifetime in order to reduce the orbital lifetime when this one exceeds 25 years.

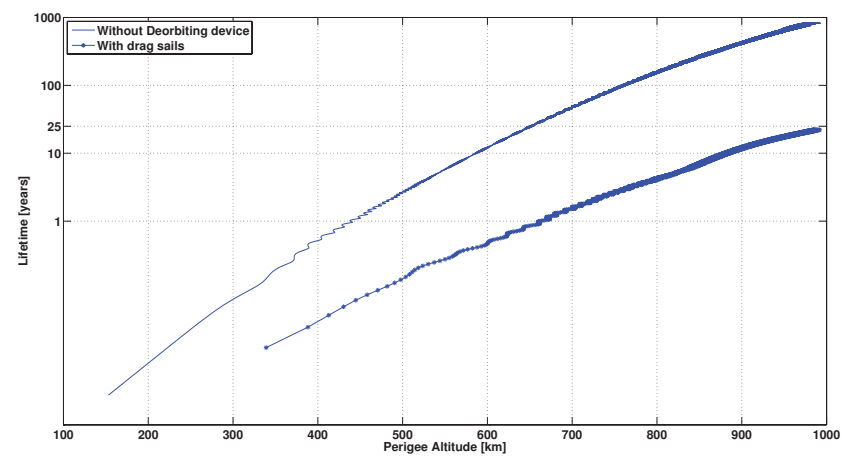

Fig. A.1. CubeSat orbital lifetime (*) without a deorbiting device and (-) with a deorbiting device, example: drag sails.

Table A.1 summarizes the parameters used in the estimation of the orbital lifetime of a $1 \mathrm{U}$ CubeSat.

Table A.1. Lifetime estimation parameters

\begin{tabular}{lll}
\hline & Parameter & Value \\
\hline Satellite & Mass & $1 \mathrm{~kg}$ \\
& Reflecting coefficient & 1.5 \\
& Reflecting area & $1.01 \mathrm{dm}^{2}$ \\
& Drag area & $1.01 \mathrm{dm}^{2}$ \\
\hline Drag sails & Drag area & $25 \mathrm{dm}^{2}$ \\
\hline Atmospheric drag & Quadrature points & 33 \\
& Recompute every & $2 \mathrm{steps}$ \\
\hline Solar radiation pressure & Quadrature points & 11 \\
\hline Simulation & Re-entry altitude & $120 \mathrm{~km}$ \\
& Integrator step & 24 hours \\
\hline
\end{tabular}

